\title{
Local existence and uniqueness for a fractional SIRS model with Mittag-Leffler law
}

\author{
Moulay Rchid Sidi Ammia,*, Mostafa Tahirib ${ }^{\mathrm{b}}$, Delfim F. M. Torres ${ }^{\mathrm{c}}$ \\ ${ }^{a}$ Department of Mathematics, AMNEA Group, Faculty of Sciences and Techniques, \\ Moulay Ismail University of Meknes, B. P. 509, Errachidia, Morocco. \\ ${ }^{b}$ Department of Mathematics, AMNEA Group, Faculty of Sciences and Techniques, \\ Moulay Ismail University of Meknes, B. P. 509, Errachidia, Morocco. \\ ${ }^{c}$ Center for Research and Development in Mathematics and Applications (CIDMA), \\ Department of Mathematics, University of Aveiro, 3810-193 Aveiro, Portugal.
}

\begin{abstract}
In this paper, we study an epidemic model with Atangana-Baleanu-Caputo fractional derivative. We obtain a special solution using an iterative scheme via Laplace transformation. Uniqueness and existence of a solution using the Banach fixed point theorem are studied. A detailed analysis of the stability of the special solution is presented. Finally, our generalized model in the derivative sense is solved numerically by the Adams-Bashforth-Moulton method.
\end{abstract}

Keywords: Epidemic model, Atangana-Baleanu-Caputo fractional derivative, Fixed point theorem, Numerical simulations. 2010 MSC: 34A08, 47H10, 26A33, 34K28.

\section{Introduction}

The generalization of mathematical models in epidemiology has for the purpose providing a good description closer to reality by using the concept of derivatives or more concretely the concept of derivatives with fractional order. Since the fractional order can be any positive real $\alpha$, one can choose the one that better fits available data [1]. Therefore, we can adjust the model to real data for better predict the future evolution of the disease [30,34]. Moreover, virus propagation is typically discontinuous and some classical differential models cannot describe it in a proper way. In contrast, fractional systems deal naturally with such discontinuous properties [16, 31].

The virus propagation is similar to heat transmission or moistness penetrability in a porous medium, which can be exactly modelled by fractional calculus [26, 39]. Authors in [20, 21] gave a geometrical description of fractional calculus, concluding that the fractional order can be related with the fractal dimension. The relationship between fractal dimension and fractional calculus has been reported by several different authors: See [29, 35] and references therein. The fractional complex transform $[22,25]$

\footnotetext{
*Corresponding author

Email addresses: rachidsidiammi@yahoo.fr (Moulay Rchid Sidi Ammi), my.mustafa.tahiri@gmail.com (Mostafa Tahiri ), delfim@ua.pt (Delfim F. M. Torres)
}

doi:10.31559/glm2021.10.2.7 
is an approximate transform of a fractal space (time) to a continuous one, and it is now widely used in fractional calculus $[10,33,36]$.

Several definitions were proposed in the literature. Some authors have used the Caputo fractional derivative because of some useful properties provided by this derivative [24, 6, 38, 12], especially in the analysis of the spread of diseases [28, 2, 3, 18]. However, this derivative has some limitations for instance the kernel therein has a singularity. To solve this problem, Caputo and Fabrizio has proposed a derivative with fractional order that has a kernel with no singularity. Recenlty the derivative was used by few researchers to solve some real world problems, see for example [27, 4]. However, many other researchers testified that the Caputo-Fabrizio operator is nothing more than a filter with a fractional regulator. They based their argument upon the fact that the kernel used in this design is local and the associate integral is the average of the given function and its integral. In many solutions of the fractional differentiation based on the power law $x^{-\sigma}$, the Mittag-Leffler function is mostly present. The Mittag-Leffler function is of course a generalization of the exponential function. In addition, it is also a non-local kernel $[5,8,9]$. To solve the failures of the Caputo-Fabrizio derivative, the fractional derivative based on the Mittag-Leffler function was introduced and used in some new problems with great success [23, 41, 40, 19]. It is important to know that where the power based on $x^{-\sigma}$ function relax then the Mittag-Leffler function raise more complex problems.

Several research papers have been published using this new concept of fractional differentiation with Mittag-Leffler function. The results obtained in [5] revealed that, the new concept is more adequate for modeling real world problems to take into account the non-locality and also to have a memory effect.

Some infectious diseases confer temporally acquired immunity. This type of disease can be modeled by the SIRS model. The total population $\mathrm{N}$ is divided into three compartments with $\mathrm{N}=\mathrm{S}+\mathrm{I}+\mathrm{R}$, where $\mathrm{S}$ is the number of susceptible, I is the number of infectious individuals and $\mathrm{R}$ is the number of individuals recovered [32].

The aim of this work is to show, by applying the Picard iteration method, the existence and uniqueness of the solution to the Atangana-Baleanu-Caputo fractional derivative for a SIRS epidemic model with saturated treatment and an incidence function presented in [32] and generalizing the classical bilinear incidence rate, the saturated incidence rate, the Beddington-DeAngelis functional response, introduced in $[15,11]$ and the Crowley-Martin functional response given in [14].

Picard iteration has more theoretical value than practical one. Finding approximate solution using this method is almost impractical for complicated function of second member of a fractional differential equation. For that, in this work we will use a numerical method called Adams-Bashforth-Moulton [7].

The paper is organized as follows. In Section 2, we give the definitions of the new fractional derivative with non-singular and non-local kernel. The existence of solution for our epidemic model via PicardLindelöf method is investigated in Section 3. In Section 4, we will study the stability analysis of the numerical scheme obtained by Picard's iteration method. Finally, in Section 5, some numerical results obtained at different instances of fractional order are presented.

\section{Basic properties of new fractional derivative}

In this section, we present the definitions of the new fractional derivative with non-singular and nonlocal kernel ([5]).

Definition 2.1. Let $f \in H^{1}(0, T), T>0,0 \leqslant \alpha<1$ then, the Atangana-Baleanu fractional derivative in Caputo sense is given as:

$$
{ }_{0}^{A B C} D_{t}^{\alpha}(f(t))=\frac{B(\alpha)}{1-\alpha} \int_{0}^{t} f^{\prime}(s) E_{\alpha}\left[-\alpha \frac{(t-s)^{\alpha}}{1-\alpha}\right] d s .
$$

where the kernel $E_{\alpha}$ is the Mittag-Leffler function of one parameter and $B(\alpha)$ is a normalization function such that $B(0)=B(1)=1([13])$. 
Definition 2.2. The fractional integral of order $\alpha$ of a new fractional derivative is defined as:

$$
{ }_{0}^{A B} I_{t}^{\alpha}(f(t))=\frac{1-\alpha}{B(\alpha)} f(t)+\frac{\alpha}{B(\alpha) \Gamma(\alpha)} \int_{0}^{t} f(u)(t-u)^{\alpha-1} d u
$$

Here $\Gamma($.$) is the Euler Gamma function which is defined as \Gamma(x)=\int_{0}^{\infty} t^{x-1} e^{-t} d t$.

Remark 2.3. When $\alpha=0$, the initial function is obtained and when $\alpha=1$, the ordinary integral is obtained.

\section{Existence and uniqueness of solution}

In this section, we extend the SIRS model to a fractional-order model, and we shall recall that the reason for the extension has been presented in the introduction. Nevertheless, it is important noting that the concept of local derivative that is used to describe the rate of change has failed to model accurately some complex real-world problems. Due to this failure, the concept of fractional differentiation based on the convolution of $x^{-\sigma}$ was introduced, and also failed in some cases due to the disc of convergence of this function. The Mittag-Leffler function, that is the more generalized version, can therefore be used in order to handle more physical problems.

We consider the following system

$$
\begin{aligned}
& { }_{0}^{A B C} D_{t}^{\alpha} S(t)=\Lambda-\mu S(t)-\frac{\beta S(t) I(t)}{1+k_{1} S(t)+k_{2} I(t)+k_{3} S(t) I(t)}+\lambda R(t), \\
& { }_{0}^{A B C} D_{t}^{\alpha} I(t)=\frac{\beta S(t) I(t)}{1+k_{1} S(t)+k_{2} I(t)+k_{3} S(t) I(t)}-(\mu+d+r) I(t)-\frac{\xi I(t)}{1+\gamma I(t)}, \\
& { }_{0}^{A B C} D_{t}^{\alpha} R(t)=r I(t)+\frac{\xi I(t)}{1+\gamma I(t)}-(\mu+\lambda) R(t),
\end{aligned}
$$

subject to initial conditions:

$$
S(0) \geqslant 0, \quad I(0) \geqslant 0, \quad R(0) \geqslant 0 .
$$

The positive constants $\Lambda, \beta, \mu, r, d, \lambda$ are the recruitment rate of the population, the infection rate, the natural death rate, the recovery rate of the infective individuals, the death rate due to disease, the rate that recovered individuals lose immunity and return to the susceptible class, respectively. While contacting with infected individuals, the susceptibles become infected at the incidence rate $\beta S I /\left(1+k_{1} S+k_{2} I+k_{3} S I\right)$, with $k_{1}, k_{2}$ and $k_{3}$ non-negative constants [32]. Through treatment, the infected individuals recover at a saturated treatment function $\xi \mathrm{I}(\mathrm{t}) /(1+\gamma \mathrm{I}(\mathrm{t}))$, where $\xi$ is positive and $\gamma$ is nonnegative and $1 /(1+\gamma \mathrm{I}(\mathrm{t}))$ describes the reverse effect of the infected being delayed for treatment. When $\gamma=0$, the saturated treatment function stays to the linear one [37].

\subsection{Iterative scheme with Laplace transform}

Theorem 3.1. For $\alpha \in[0,1]$, the following time fractional ordinary differential equation

$$
{ }_{0}^{A B C} D_{t}^{\alpha}(f(t))=u(t),
$$

has a unique solution, namely

$$
f(t)=f(0)+\frac{1-\alpha}{B(\alpha)} u(t)+\frac{\alpha}{B(\alpha) \Gamma(\alpha)} \int_{0}^{t} u(p)(t-p)^{\alpha-1} d p
$$


Proof. Using the Laplace transformation on both sides of the equation (3.3):

$$
L\left[\int_{0}^{A B C} D_{t}^{\alpha}(f(t))\right](p)=L[u(t)](p), \quad p>0
$$

and according to [8, Theorem 3], we have that

$$
\frac{B(\alpha)}{1-\alpha} \frac{p^{\alpha} L\{f(t)\}(p)-p^{\alpha-1} f(0)}{p^{\alpha}+\frac{\alpha}{1-\alpha}}=L\{u(t)\}(p),
$$

which is equivalent,

$$
\mathrm{L}\{\mathrm{f}(\mathrm{t})\}(\mathrm{p})=\frac{1}{\mathrm{p}} \mathbf{f}(0)+\frac{1-\alpha}{B(\alpha)} \mathrm{L}\{\mathrm{u}(\mathrm{t})\}(\mathrm{p})+\frac{\alpha}{\mathrm{p}^{\alpha} \mathrm{B}(\alpha)} \mathrm{L}\{\mathrm{u}(\mathrm{t})\}(\mathrm{p}) .
$$

Now, we use the inverse Laplace transform

$$
f(t)=f(0)+\frac{1-\alpha}{B(\alpha)} u(t)+L^{-1}\left\{\frac{\alpha}{p^{\alpha} B(\alpha)} L\{u(t)\}(p)\right\}(t) .
$$

We have

$$
\frac{\alpha}{p^{\alpha} B(\alpha)}=\frac{\alpha}{B(\alpha)} L\left\{\frac{t^{\alpha-1}}{\Gamma(\alpha)}\right\}(p)
$$

Let $F(p)=\frac{\alpha}{B(\alpha)} L\left\{\frac{t^{\alpha-1}}{\Gamma(\alpha)}\right\}(p)$ and $G(p)=L\{u(t)\}(p)$, then applying the convolution theorem, we obtain

$$
\begin{aligned}
L^{-1}\left\{\frac{\alpha}{p^{\alpha} B(\alpha)} L\{u(t)\}(p)\right\}(t) & =L^{-1}\{F(p) \times G(p)\}(t)=\frac{\alpha}{B(\alpha)}\left(u(t) * \frac{t^{\alpha-1}}{\Gamma(\alpha)}\right)(p) \\
& =\frac{\alpha}{B(\alpha) \Gamma(\alpha)} \int_{0}^{t} u(p)(t-p)^{\alpha-1} d p .
\end{aligned}
$$

Hence the result.

Using the above theorem, our system is equivalent to the following

$$
\begin{aligned}
S(t)-S(0)= & \frac{1-\alpha}{B(\alpha)}\left\{\Lambda-\mu S(t)-\frac{\beta S(t) I(t)}{1+k_{1} S(t)+k_{2} I(t)+k_{3} S(t) I(t)}+\lambda R(t)\right\} \\
& +\frac{\alpha}{B(\alpha) \Gamma(\alpha)} \int_{0}^{t}(t-p)^{\alpha-1}\{\Lambda-\mu S(p) \\
& \left.-\frac{\beta S(p) I(p)}{1+k_{1} S(p)+k_{2} I(p)+k_{3} S(p) I(p)}+\lambda R(p)\right\} d p, \\
I(t)-I(0)= & \frac{1-\alpha}{B(\alpha)}\left\{\frac{\beta S(t) I(t)}{1+k_{1} S(t)+k_{2} I(t)+k_{3} S(t) I(t)}-(\mu+d+r) I(t)\right. \\
& \left.-\frac{\xi I(t)}{1+\gamma I(t)}\right\}-\frac{\alpha}{B(\alpha) \Gamma(\alpha)} \int_{0}^{t}(t-p)^{\alpha-1}\{(\mu+d+r) I(p) \\
& \left.+\frac{\xi I(p)}{1+\gamma I(p)}-\frac{\beta S(p) I(p)}{1+k_{1} S(p)+k_{2} I(p)+k_{3} S(p) I(p)}\right\} d p, \\
R(t)-R(0)= & \frac{1-\alpha}{B(\alpha)}\left\{r I(t)+\frac{\xi I(t)}{1+\gamma I(t)}-(\mu+\lambda) R(t)\right\} \\
& +\frac{\alpha}{B(\alpha) \Gamma(\alpha)} \int_{0}^{t}(t-p)^{\alpha-1}\left\{r I(p)+\frac{\xi I(p)}{1+\gamma I(p)}-(\mu+\lambda) R(p)\right\} d p .
\end{aligned}
$$


The iterative scheme of the system (3.5) is given by:

$$
\begin{aligned}
& S_{0}(t)=S(0) ; \quad I_{0}(t)=I(0) ; \quad R_{0}(t)=R(0) . \\
& S_{n+1}(t)= \frac{1-\alpha}{B(\alpha)}\left\{\Lambda-\mu S_{n}(t)-\frac{\beta S_{n}(t) I_{n}(t)}{1+k_{1} S_{n}(t)+k_{2} I_{n}(t)+k_{3} S_{n}(t) I_{n}(t)}+\lambda R_{n}(t)\right\} \\
&+\frac{\alpha}{B(\alpha) \Gamma(\alpha)} \int_{0}^{t}(t-p)^{\alpha-1}\left\{\Lambda-\mu S_{n}(p)\right. \\
&\left.-\frac{\beta S_{n}(p) I_{n}(p)}{1+k_{1} S_{n}(p)+k_{2} I_{n}(p)+k_{3} S_{n}(p) I_{n}(p)}+\lambda R_{n}(p)\right\} d p \\
& I_{n+1}(t)= \frac{1-\alpha}{B(\alpha)}\left\{\frac{\beta S_{n}(t) I_{n}(t)}{1+k_{1} S_{n}(t)+k_{2} I_{n}(t)+k_{3} S_{n}(t) I_{n}(t)}-(\mu+d+r) I_{n}(t)\right. \\
&\left.-\frac{\xi I_{n}(t)}{1+\gamma I_{n}(t)}\right\}-\frac{\alpha}{B(\alpha) \Gamma(\alpha)} \int_{0}^{t}(t-p)^{\alpha-1}\left\{(\mu+d+r) I_{n}(p)\right. \\
&\left.+\frac{\xi I_{n}(p)}{1+\gamma I_{n}(p)}-\frac{\beta S_{n}(p) I_{n}(p)}{1+k_{1} S_{n}(p)+k_{2} I_{n}(p)+k_{3} S_{n}(p) I_{n}(p)}\right\} d p, \\
& R_{n+1}(t)= \frac{1-\alpha}{B(\alpha)}\left\{r I_{n}(t)+\frac{\xi I_{n}(t)}{1+\gamma I_{n}(t)}-(\mu+\lambda) R_{n}(t)\right\} \\
&+\frac{\alpha}{B(\alpha) \Gamma(\alpha)} \int_{0}^{t}(t-p)^{\alpha-1}\left\{r I_{n}(p)+\frac{\xi I_{n}(p)}{1+\gamma I_{n}(p)}-(\mu+\lambda) R_{n}(p)\right\} d p .
\end{aligned}
$$

If we take the limit with greater than $n$, we expect to obtain the exact solution.

\subsection{Existence of solution via Picard-Lindelöf method}

We define the following operators:

$$
\begin{aligned}
& f_{1}(t, \Omega(t))=\Lambda-\mu S(t)-\frac{\beta S(t) I(t)}{1+k_{1} S(t)+k_{2} I(t)+k_{3} S(t) I(t)}+\lambda R(t), \\
& f_{2}(t, \Omega(t))=\frac{\beta S(t) I(t)}{1+k_{1} S(t)+k_{2} I(t)+k_{3} S(t) I(t)}-(\mu+d+r) I(t)-\frac{\xi I(t)}{1+\gamma I(t)}, \\
& f_{3}(t, \Omega(t))=r I(t)+\frac{\xi I(t)}{1+\gamma I(t)}-(\mu+\lambda) R(t),
\end{aligned}
$$

and the matrix form of system (3.1) subject to conditions (3.2) :

$$
{ }_{0}^{A B C} D_{t}^{\alpha} \Omega(t)=F(t, \Omega(t)), \quad \Omega(0)=\Omega_{0},
$$

where $\Omega(t)=(S(t), I(t), R(t)), \Omega_{0}=\left(S_{0}, I_{0}, R_{0}\right)$ and $F(t, \Omega(t))=\left(f_{1}(t, \Omega(t)), f_{2}(t, \Omega(t)), f_{3}(t, \Omega(t))\right)$.

Lemma 3.2. The function $\mathrm{F}$ is Lipschitz continuous on $[0, \mathrm{~T}] \times \mathbf{B}\left(\Omega_{0}, \rho\right)$, with

$$
[0, T] \times \mathbf{B}\left(\Omega_{0}, \rho\right)=\left\{(t, \Omega(t)) \in[0, T] \times \mathbb{R}_{+}^{3} / \sup _{t \in[0, T]}\left\|\Omega(t)-\Omega_{0}\right\|_{1} \leqslant \rho\right\},
$$

i.e., there exists a constant $\mathrm{L} \in \mathbb{R}_{+}, \forall\left(t, \Omega_{1}(t)\right),\left(t, \Omega_{2}(t)\right) \in[0, T] \times \mathbf{B}\left(\Omega_{0}, \rho\right)$

$$
\left\|F\left(t, \Omega_{1}(t)\right)-F\left(t, \Omega_{2}(t)\right)\right\|_{1} \leqslant L\left\|\Omega_{1}(t)-\Omega_{2}(t)\right\|_{1},
$$

with $\|\Omega(t)\|_{1}=\sum_{i=1}^{3}\left|\Omega_{i}(t)\right|$ is the Manhattan norm. 
Proof. We shall prove that F satisfies the Lipschitz condition in the second argument $\Omega$.

$$
\begin{aligned}
& \left\|F\left(t, \Omega_{1}(t)\right)-F\left(t, \Omega_{2}(t)\right)\right\|_{1}=\left|f_{1}\left(t, \Omega_{1}(t)\right)-f_{1}\left(t, \Omega_{2}(t)\right)\right|+\mid f_{2}\left(t, \Omega_{1}(t)\right) \\
& -f_{2}\left(t, \Omega_{2}(t)\right)|+| f_{3}\left(t, \Omega_{1}\right)-f_{3}\left(t, \Omega_{2}\right) \mid \\
& =\mid \Lambda-\mu S_{1}(t)-\frac{\beta S_{1}(t) I_{1}(t)}{1+k_{1} S_{1}(t)+k_{2} I_{1}(t)+k_{3} S_{1}(t) I_{1}(t)}+\lambda R_{1}(t) \\
& -\left(\Lambda-\mu S_{2}(t)-\frac{\beta S_{2}(t) I_{2}(t)}{1+k_{1} S_{2}(t)+k_{2} I_{2}(t)+k_{3} S_{2}(t) I_{2}(t)}+\lambda R_{2}(t)\right) \mid \\
& +\mid \frac{\beta S_{1}(t) I_{1}(t)}{1+k_{1} S_{1}(t)+k_{2} I_{1}(t)+k_{3} S_{1}(t) I_{1}(t)}-(\mu+d+r) I_{1}(t)-\frac{\xi I_{1}(t)}{1+\gamma I_{1}(t)} \\
& -\left(\frac{\beta S_{2}(t) I_{2}(t)}{1+k_{1} S_{2}(t)+k_{2} I_{2}(t)+k_{3} S_{2}(t) I_{2}(t)}-(\mu+d+r) I_{2}(t)-\frac{\xi I_{2}(t)}{1+\gamma I_{2}(t)}\right) \mid \\
& +\left|r I_{1}(t)+\frac{\xi I_{1}(t)}{1+\gamma I_{1}(t)}-(\mu+\lambda) R_{1}(t)-\left(r I_{2}(t)+\frac{\xi I_{2}(t)}{1+\gamma I_{2}(t)}-(\mu+\lambda) R_{2}(t)\right)\right| .
\end{aligned}
$$

We reduce the next two fractions to the same denominator

$$
D=\left(1+k_{1} S_{1}(t)+k_{2} I_{1}(t)+k_{3} S_{1}(t) I_{1}(t)\right)\left(1+k_{1} S_{2}(t)+k_{2} I_{2}(t)+k_{3} S_{2}(t) I_{2}(t)\right) .
$$

We note that $\mathrm{D}>1$, hence:

$$
\begin{aligned}
& \mid \frac{\beta S_{1}(t) I_{1}(t)}{1+}+\frac{\beta S_{2}(t) I_{2}(t)}{1+(t)+k_{2} I_{1}(t)+k_{3} S_{1}(t) I_{1}(t)}-\frac{k_{1} S_{2}(t)+k_{2} I_{2}(t)+k_{3} S_{2}(t) I_{2}(t)}{} \\
& \quad \leqslant \beta\left|S_{1}(t) I_{1}(t)-S_{2}(t) I_{2}(t)\right|+\beta k_{1}\left|S_{1}(t)\right|\left|S_{2}(t) \| I_{1}(t)-I_{2}(t)\right| \\
& \quad+\beta k_{2}\left|I_{1}(t)\right|\left|I_{2}(t)\right|\left|S_{1}(t)-S_{2}(t)\right| \\
& \quad \leqslant \beta\left|S_{1}(t) I_{1}(t)-S_{2}(t) I_{1}(t)\right|+\beta\left|S_{2}(t) I_{1}(t)-S_{2}(t) I_{2}(t)\right| \\
& \quad+\beta k_{1}\left|S_{1}(t)\right|\left|S_{2}(t)\right|\left|I_{1}(t)-I_{2}(t)\right|+\beta k_{2}\left|I_{1}(t)\right|\left|I_{2}(t)\right|\left|S_{1}(t)-S_{2}(t)\right| \\
& \quad \leqslant \beta\left|I_{2}(t)\right|\left|S_{1}(t)-S_{2}(t)\right|+\beta\left|S_{1}(t) \| I_{1}(t)-I_{2}(t)\right| \\
& \quad+\beta k_{1}\left|S_{1}(t)\right|\left|S_{2}(t)\right|\left|I_{1}(t)-I_{2}(t)\right|+\beta k_{2}\left|I_{1}(t)\right|\left|I_{2}(t)\right|\left|S_{1}(t)-S_{2}(t)\right| .
\end{aligned}
$$

On the same manner, we can prove that

$$
\left|\frac{\xi \mathrm{I}_{1}(\mathrm{t})}{1+\gamma \mathrm{I}_{1}(\mathrm{t})}-\frac{\xi \mathrm{I}_{2}(\mathrm{t})}{1+\gamma \mathrm{I}_{2}(\mathrm{t})}\right| \leqslant \xi\left|\mathrm{I}_{1}(\mathrm{t})-\mathrm{I}_{2}(\mathrm{t})\right| .
$$

Then

$$
\begin{aligned}
& \left\|F\left(t, \Omega_{1}(t)\right)-F\left(t, \Omega_{2}(t)\right)\right\|_{1} \\
& \leqslant \mu\left|S_{1}(t)-S_{2}(t)\right|+\lambda\left|R_{1}(t)-R_{2}(t)\right|+\beta\left|I_{2}(t)\right|\left|S_{1}(t)-S_{2}(t)\right| \\
& +\beta\left|S_{1}(t)\right|\left|I_{1}(t)-I_{2}(t)\right| \\
& +\beta k_{1}\left|S_{1}(t)\right|\left|S_{2}(t)\right|\left|I_{1}(t)-I_{2}(t)\right|+\beta k_{2}\left|I_{1}(t)\right|\left|I_{2}(t)\right|\left|S_{1}(t)-S_{2}(t)\right| \\
& +\beta\left|I_{2}(t)\right|\left|S_{1}(t)-S_{2}(t)\right|+\beta\left|S_{1}(t)\right|\left|I_{1}(t)-I_{2}(t)\right| \\
& +\beta k_{1}\left|S_{1}(t)\right|\left|S_{2}(t)\right|\left|I_{1}(t)-I_{2}(t)\right|+\beta k_{2}\left|I_{1}(t)\right|\left|I_{2}(t)\right|\left|S_{1}(t)-S_{2}(t)\right| \\
& +(\mu+d+r)\left|I_{1}(t)-I_{2}(t)\right|+\xi\left|I_{1}(t)-I_{2}(t)\right| \\
& +r\left|I_{1}(t)-I_{2}(t)\right|+(\mu+\lambda)\left|R_{1}(t)-R_{2}(t)\right|+\xi\left|I_{1}(t)-I_{2}(t)\right| \\
& \leqslant\left(\mu+2 \beta\left|I_{2}(t)\right|+2 \beta k_{2}\left|I_{1}(t) \| I_{2}(t)\right|\right)\left|S_{1}(t)-S_{2}(t)\right| \\
& +\left(2 \beta\left|S_{1}(t)\right|+2 \beta k_{1}\left|S_{1}(t)\right|\left|S_{2}(t)\right|+\mu+d+2 r+2 \xi\right)\left|I_{1}(t)-I_{2}(t)\right| \\
& +(2 \lambda+\mu)\left|R_{1}(t)-R_{2}(t)\right|
\end{aligned}
$$




$$
\begin{aligned}
& \leqslant\left(\mu+2 \beta\left(\rho+I_{0}\right)+2 \beta k_{2}\left(\rho+I_{0}\right)^{2}\right)\left|S_{1}(t)-S_{2}(t)\right|+\left(2 \beta\left(\rho+S_{0}\right)\right. \\
& \left.+2 \beta k_{1}\left(\rho+S_{0}\right)^{2}+\mu+d+2 r+2 \xi\right)\left|I_{1}(t)-I_{2}(t)\right|+(2 \lambda+\mu)\left|R_{1}(t)-R_{2}(t)\right| \\
& \leqslant L\left\|\Omega_{1}(t)-\Omega_{2}(t)\right\|_{1}
\end{aligned}
$$

where

$$
\begin{gathered}
\mathrm{L}=\operatorname{Max}\left\{\left(\mu+2 \beta\left(\rho+\mathrm{I}_{0}\right)+2 \beta \mathrm{k}_{2}\left(\rho+\mathrm{I}_{0}\right)^{2}\right),\left(2 \beta\left(\rho+\mathrm{S}_{0}\right)+2 \beta \mathrm{k}_{1}\left(\rho+\mathrm{S}_{0}\right)^{2}+\mu\right.\right. \\
+\mathrm{d}+2 \mathrm{r}+2 \xi),(2 \lambda+\mu)\} .
\end{gathered}
$$

It is clear that $L>0$, then $F$ is Lipschitz continuous in the second argument.

Theorem 3.3. Let $W=\left\{\Omega \in\left(C^{0}[0, \delta]\right)^{3}: \Omega(0)=\Omega_{0}, \Omega(t) \in \mathbf{B}\left(\Omega_{0}, \rho\right)\right\}$ be the space of continuous functions which is complete with norm $\|\Omega\|_{w}=\sup _{t \in[0, \delta]}\|\Omega(t)\|_{1}$. Let $\|\mathrm{F}(\mathrm{t}, \Omega)\|_{\mathrm{w}} \leqslant \overline{\mathrm{N}}$ for $(\mathrm{t}, \Omega) \in[0, \delta] \times \mathbf{B}\left(\Omega_{0}, \rho\right)$. Then the system (3.1) subject to conditions (3.2) has a unique solution on $[0, \delta]$ with $\delta>0$ and

$$
\delta<\min \left\{\mathrm{T},\left(\frac{\rho \mathrm{B}(\alpha) \Gamma(\alpha)}{\bar{N}}+\alpha \Gamma(\alpha)-\Gamma(\alpha)\right)^{\alpha^{-1}},\left(\frac{\mathrm{B}(\alpha) \Gamma(\alpha)}{\mathrm{L}}+\alpha \Gamma(\alpha)-\Gamma(\alpha)\right)^{\alpha^{-1}}\right\}
$$

Proof. The fixed-point theorem in Banach space $W$ can be employed here. For that, the Picard's operator $\Theta$ is defined between the functional space $W$ into itself, as follows:

$$
\Theta: W \rightarrow W
$$

such that

$$
\Theta \Omega(t)=\Omega_{0}+\frac{1-\alpha}{B(\alpha)} F(t, \Omega(t))+\frac{\alpha}{B(\alpha) \Gamma(\alpha)} \int_{0}^{t} F(p, \Omega(p))(t-p)^{\alpha-1} d p
$$

We show that $\Theta$ maps $W$ to $W$. Due to the fact that there is no disease that is able to kill the whole world population, we can suppose that $\Omega(t) \in W$. We can also assume that the solution is bounded within a period of time since the number of targeted population is finite. Next, we show that $\Theta \Omega(t) \in W$.

$$
\begin{aligned}
\left\|\Theta \Omega-\Omega_{0}\right\|_{w}= & \sup _{t \in[0, \delta]}\left\|\frac{1-\alpha}{B(\alpha)} F(t, \Omega(t))+\frac{\alpha}{B(\alpha) \Gamma(\alpha)} \int_{0}^{t} F(p, \Omega(p))(t-p)^{\alpha-1} d p\right\|_{1} \\
\leqslant & \frac{1-\alpha}{B(\alpha)} \times \sup _{t \in[0, \delta]}\|F(t, \Omega(t))\|_{1}+\frac{\alpha}{B(\alpha) \Gamma(\alpha)} \\
& \times \sup _{t \in[0, \delta]} \int_{0}^{t}\|F(p, \Omega(p))\|_{1}(t-p)^{\alpha-1} d p \\
\leqslant & \frac{1-\alpha}{B(\alpha)} \times \sup _{t \in[0, \delta]}\|F(t, \Omega(t))\|_{1}+\frac{\alpha}{B(\alpha) \Gamma(\alpha)} \\
& \times \int_{0}^{\delta} \sup _{p \in[0, \delta]}\|F(p, \Omega(p))\|_{1}(t-p)^{\alpha-1} d p \\
\leqslant & \frac{(1-\alpha)}{B(\alpha)} \bar{N}+\frac{\alpha}{B(\alpha) \Gamma(\alpha)} \bar{N} \int_{0}^{\delta}(t-p)^{\alpha-1} d p \\
\leqslant & \frac{(1-\alpha)}{B(\alpha)} \bar{N}+\frac{\alpha}{B(\alpha) \Gamma(\alpha)} \bar{N} \int_{0}^{\delta}(t-p)^{\alpha-1} d p \\
\leqslant & \frac{(1-\alpha)}{B(\alpha)} \bar{N}+\frac{\delta^{\alpha}}{B(\alpha) \Gamma(\alpha)} \bar{N} .
\end{aligned}
$$


Then, it is necessary that $\frac{(1-\alpha)}{B(\alpha)} \bar{N}+\frac{\delta^{\alpha}}{B(\alpha) \Gamma(\alpha)} \bar{N} \leqslant \rho$, this implies :

$$
\delta<\left(\frac{\rho B(\alpha) \Gamma(\alpha)}{\bar{N}}+\alpha \Gamma(\alpha)-\Gamma(\alpha)\right)^{\alpha^{-1}}
$$

for $\Theta$ maps $W$ into itself.

Using the definition of the operator defined in (3.17), we deduce the following

$$
\begin{aligned}
& \left\|\Theta \Omega_{1}-\Theta \Omega_{2}\right\| w=\sup _{t \in[0, \delta]} \| \frac{1-\alpha}{B(\alpha)}\left\{F\left(t, \Omega_{1}(t)\right)-F\left(t, \Omega_{2}(t)\right)\right\} \\
& \quad+\frac{\alpha}{B(\alpha) \Gamma(\alpha)} \int_{0}^{t}\left\{F\left(p, \Omega_{1}(p)\right)-F\left(p, \Omega_{2}(p)\right)\right\}(t-p)^{\alpha-1} d p \|_{1} \\
& \leqslant \frac{1-\alpha}{B(\alpha)} \times \sup _{t \in[0, \delta]}\left\|F\left(t, \Omega_{1}(t)\right)-F\left(t, \Omega_{2}(t)\right)\right\|_{1} \\
& \quad+\frac{\alpha}{B(\alpha) \Gamma(\alpha)} \times \sup _{t \in[0, \delta]} \int_{0}^{t}\left\|F\left(p, \Omega_{1}(p)\right)-F\left(p, \Omega_{2}(p)\right)\right\|_{1}(t-p)^{\alpha-1} d p .
\end{aligned}
$$

In Lemma 3.2, we have shown that F is Lipschitzian with respect to the second argument. Then,

$$
\begin{aligned}
&\left\|\Theta \Omega_{1}-\Theta \Omega_{2}\right\| w \leqslant \frac{(1-\alpha) \mathrm{L}}{\mathrm{B}(\alpha)} \times \sup _{\mathrm{t} \in[0, \delta]}\left\|\Omega_{1}(\mathrm{t})-\Omega_{2}(\mathrm{t})\right\|_{1} \\
& \quad+\frac{\alpha \mathrm{L}}{\mathrm{B}(\alpha) \Gamma(\alpha)} \int_{0}^{\delta}\left\|\Omega_{1}(p)-\Omega_{2}(p)\right\|_{1}(\mathrm{t}-\mathrm{p})^{\alpha-1} \mathrm{~d} p \\
& \leqslant \frac{(1-\alpha) \mathrm{L}}{\mathrm{B}(\alpha)} \times \sup _{\mathrm{t} \in[0, \delta]}\left\|\Omega_{1}(\mathrm{t})-\Omega_{2}(\mathrm{t})\right\|_{1} \\
&+\frac{\alpha \mathrm{L}}{\mathrm{B}(\alpha) \Gamma(\alpha)} \int_{0}^{\delta} \sup _{p \in[0, \delta]}\left\|\Omega_{1}(p)-\Omega_{2}(p)\right\|_{1}(\mathrm{t}-\mathrm{p})^{\alpha-1} \mathrm{dp} \\
& \leqslant \frac{(1-\alpha) \mathrm{L}}{\mathrm{B}(\alpha)}\left\|\Omega_{1}-\Omega_{2}\right\|_{W}+\frac{\alpha \mathrm{L}}{\mathrm{B}(\alpha) \Gamma(\alpha)} \int_{0}^{\delta}\left\|\Omega_{1}-\Omega_{2}\right\|_{W}(t-p)^{\alpha-1} \mathrm{dp} \\
& \leqslant\left(\frac{(1-\alpha) \mathrm{L}}{\mathrm{B}(\alpha)}+\frac{\delta^{\alpha} \mathrm{L}}{\mathrm{B}(\alpha) \Gamma(\alpha)}\right)\left\|\Omega_{1}-\Omega_{2}\right\|_{W} .
\end{aligned}
$$

Thus, the defined operator $\Theta$ is a contraction with a unique fixed point $\Omega \in W$ if $L\left(\frac{(1-\alpha)}{B(\alpha)}+\frac{\delta^{\alpha}}{B(\alpha) \Gamma(\alpha)}\right)<$ 1 which is implies that

$$
\delta<\left(\frac{\mathrm{B}(\alpha) \Gamma(\alpha)}{\mathrm{L}}+\alpha \Gamma(\alpha)-\Gamma(\alpha)\right)^{\alpha^{-1}} .
$$

This shows that the system under investigation has a unique solution.

\section{Stability analysis of Picard's iteration method}

In the following we study the stability of our iterative scheme proposed in (3.7) to show its convergence.

Definition 4.1. (see[42]) Let $(X,\|\|$.$) be a Banach space and \Theta$ a self-map of $X$. Let $y_{n+1}=g\left(\Theta, y_{n}\right)$ be particular recursive procedure. Suppose that $F_{P}(\Theta)$ is the fixed-point set of $\Theta$ and has at least one element and that $y_{n}$ converges to a point $p \in F_{P}(\Theta)$. Let $\left\{x_{n}\right\} \subseteq X$ and define $e_{n}=\left\|x_{n+1}-g\left(\Theta, x_{n}\right)\right\|$. If $\lim _{n \rightarrow+\infty} e_{n}=0$ implies that $\lim _{n \rightarrow+\infty} x_{n}=p$, then the iteration method $y_{n+1}=g\left(\Theta, y_{n}\right)$ is said to be $\Theta$-stable. 
Remark 4.2. (see[42]) Without any loss of generality, we must assume that $\left\{x_{n}\right\}$ is bounded. Otherwise, if $\left\{x_{n}\right\}$ is not bounded, then it cannot converge. If all conditions in Definition 4.1 are satisfied for $y_{n+1}=$ $\Theta\left(y_{n}\right)$ which is known as Picard's iteration, consequently, the iteration will be $\Theta$-stable.

We shall then state the following theorem.

Theorem 4.3. (see[42]) Let $(X,\|\|$.$) be a Banach space and \Theta$ a self-map of $X$ satisfying

$$
\|\Theta(x)-\Theta(y)\| \leqslant K\|\Theta(x)-x\|+k\|x-y\|
$$

for all $x, y$ in $\mathrm{X}$ where $0 \leqslant \mathrm{~K}, 0 \leqslant \mathrm{k}<1$. Suppose that $\Theta$ has a fixed point. Then, $\Theta$ is Picard's $\Theta$-stable.

Theorem 4.4. The system (3.7) is $\Theta$-stable in Banach space $W$ if it satisfies the condition (3.22).

Proof. Let $n, m \in \mathbb{N}$, we put $\Omega_{n}=\left(S_{n}, I_{n}, R_{n}\right)$ and $\Theta\left(\Omega_{n}\right)=\left(S_{n+1}, I_{n+1}, R_{n+1}\right)$. In order to show that $P$ admits a fixed point, we introduce the norm on both sides and we have

$$
\begin{gathered}
\left\|\Theta\left(\Omega_{n}(t)\right)-\Theta\left(\Omega_{m}(t)\right)\right\|_{1} \leqslant \frac{1-\alpha}{B(\alpha)}\left\|F\left(t, \Omega_{n}(t)\right)-F\left(t, \Omega_{m}(t)\right)\right\|_{1} \\
+\frac{\alpha}{B(\alpha) \Gamma(\alpha)} \int_{0}^{t}\left\|F\left(t, \Omega_{n}(t)\right)-F\left(t, \Omega_{m}(t)\right)\right\|_{1}(t-p)^{\alpha-1} d p .
\end{gathered}
$$

Then, the same arguments as before lead to

$$
\left\|\Theta\left(\Omega_{n}\right)-\Theta\left(\Omega_{m}\right)\right\|_{W} \leqslant L\left(\frac{1-\alpha}{B(\alpha)}+\frac{\delta^{\alpha}}{B(\alpha) \Gamma(\alpha)}\right)\left\|\Omega_{n}-\Omega_{m}\right\|_{W}
$$

If condition (3.22) is satisfied, then the condition (4.1) of Theorem 4.3 holds. This completes the proof.

\section{Numerical Simulations}

We present numerical simulations of the special solution of our model using the Adams-BashforthMoulton method presented in [7] for different arbitrary values of fractional order $\alpha$. The convergence and stability of this method apply at very long intervals. It is a reliable and yet fast alternative for the approximate evaluation of Mittag-Leffler functions[17]. Moreover, it is very useful for the numerical evaluation of other special functions arising in fractional calculus[17]. In our numerical computations, we consider the following values of the parameters: $\Lambda=0.7, \mu=0.1, \lambda=0.02, \beta=0.1, r=0.02, d=0.01$, $\xi=0.07, \gamma=0.1, k_{1}=0.1, k_{2}=0.02$, and $k_{3}=0.002$ with initial conditions $S(0)=3.0, I(0)=2.0$, $\mathrm{R}(0)=1.0$. The numerical results given in Figure 1 show numerical simulations of the special solution of our model as a function of time for different values of $\alpha$.

Figure 1 shows the dynamics of the interactions between the compartments of the susceptible and the infected in a particular environment. We notice that when the number of susceptible people decreases the number of infected people increases.

\section{Conclusion}

Recently, the fractional derivative of Baleanu-Atangana in the sense of Caputo has been used for some applications in many scientific fields with great success. This fractional differentiation based on the Mittag-Leffler function. In this work, we extended the SIRS model with a saturated treatment and a nonlinear incidence function to the concept of fractional differentiation Atangana-Baleanu-Caputo. We have shown the existence and uniqueness of our model using the fixed point theorem. Analysis of the stability of the iterative scheme is validated via the $\Theta$-stable approach. Finally, numerical simulations presented for different values of $\alpha$.

Since our epidemic model contains saturated treatment function, then as a perspective we will study the influence of the parameter $\gamma$ on the stability of our system which leads to the case of multiple equilibrium points and shows bifurcation phenomena. 

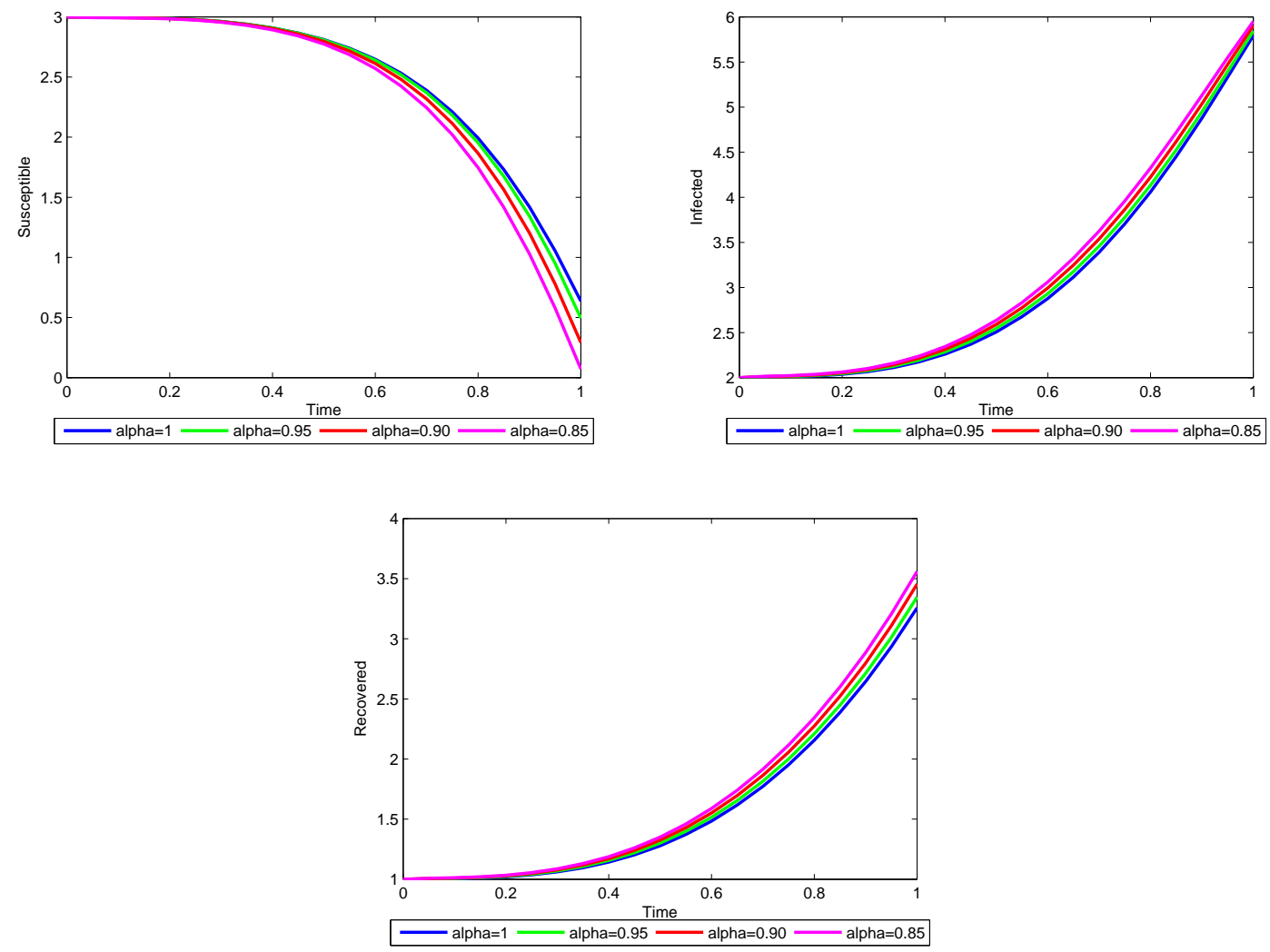

Figure 1: Nature of solution with respect to time for different values of $\alpha$.

\section{Acknowledgements}

Torres was partially funded by FCT, project UIDB/04106/2020 (CIDMA).

\section{References}

[1] R. Almeida, What is the best fractional derivative to fit data?, Appl. Anal. Discrete Math., 11 (2017), no. 2, $358-368$. https: / / doi.org/10.2298/aadm170428002a 1

[2] I. Area, H. Batarfi, J. Losada, J.J. Nieto, W. Shammakh and A. Torres, On a fractional order Ebola epidemic model, Adv. Difference Equ., 2015 (2015), no. 1, 278 pp. https:/ / doi.org/10.1186/s13662-015-0613-5 1

[3] I. Area, J. Losada, F. Ndairou, J.J. Nieto and D.D. Tcheutia, Mathematical modeling of 2014 Ebola outbreak, Math. Method Appl. Sci., 40 (2017), no. 17, 6114-6122. https:/ / doi.org/10.1002/mma.3794 1

[4] A. Atangana and B.S.T. Alkahtani, Analysis of the Keller-Segel model with a fractional derivative without singular kernel, Entropy, 17 (2015), no. 6, 4439-4453. https://doi.org/10.3390/e17064439 1

[5] A. Atangana and D. Baleanu, New fractional derivatives with nonlocal and non-singular kernel: theory and application to heat transfer model, Thermal Science, 20 (2016), no. 2, 763-769. https://doi.org/10.2298/tsci160111018a 1, 2

[6] A. Atangana and E.F.G. Doungmo, Extension of matched asymptotic method to fractional boundary layers problems, Mathematical Problems in Engineering, 2014 (2014), Article ID 107535, 7 pp, DOI:10.1155/2014/107535. https:/ / doi.org/10.1155/2014/107535 1

[7] A. Atangana and J.F. Gómez-Aguilar, Decolonisation of fractional calculus rules: Breaking commutativity and associativity to capture more natural phenomena, Eur. Phys. J. Plus, 133 (2018), no. 4, 1 pp. https://doi.org/10.1140/epjp/i2018-12021-3 1,5

[8] A. Atangana and I. Koca, Chaos in a simple nonlinear system with Atangana-Baleanu derivatives with fractional order, Chaos, Solitons and Fractals, 89 (2016), 447-454. https:/ / doi.org/10.1016/j.chaos.2016.02.012 1, 3.1

[9] A. Atangana and I. Koca, On the new fractional derivative and application to nonlinear Baggs and Freedman model, J. Nonlinear Sci. Appl., 9 (2016), 2467-2480. https:/ / doi.org/10.22436/jnsa.009.05.46 1

[10] B. Bayour and D. F. M. Torres, Complex-valued fractional derivatives on time scales, in Differential and difference equations with applications, 79-87, Springer Proc. Math. Stat., 164, Springer, Cham, 2016. https://doi.org/10.1007/978-3-319-32857-7.1 
[11] J. R. Beddington, Mutual interference between parasites or predators and its effect on searching effciency, J. Anim. Ecol., 44 (1975), 331-340. https:/ / doi.org/10.2307/3866 1

[12] M. Caputo, Linear models of dissipation whose $Q$ is almost frequency independent-part II, Geophysical Journal International, 13 (1967), no. 5, 529-539. https:/ / doi.org/10.1111/j.1365-246x.1967.tb02303.x 1

[13] M. Caputo and M. Fabrizio, A new definition of fractional derivative without singular kernel, Progress in Fractional Differentiation and Applications, 1 (2015), no. 2, 1-13. 2.1

[14] P. H. Crowley and E. K. Martin, Functional responses and interference within and between year classes of a dragonfly population, J. North Am. Benthol. Soc., 8 (1989), no. 3, 211-221. https:/ / doi.org/10.2307/1467324 1

[15] D. L. DeAngelis, R. A. Goldsten and R. V. O'Neill, A model for trophic interaction, Ecology, 56 (1975), 881-892. 1

[16] A. Debbouche, J. J. Nieto and D. F. M. Torres, Optimal solutions to relaxation in multiple control problems of Sobolev type with nonlocal nonlinear fractional differential equations, J. Optim. Theory Appl., 174 (2017), no. 1, 7-31. 1

[17] K. Diethelma, N. J. Ford and A. D. Freed, Detailed error analysis for a fractional Adams method, Numerical Algorithms, 36 (2004), no. 1, 31-52. 5

[18] G.E.F. Doungmo, Stability and convergence analysis of a variable order replicator-mutator process in a moving medium, Journal of Theoretical Biology, 403 (2016), 178-187. 1

[19] F. Gao and X-J. Yang, Fractional Maxwell fluid with fractional derivative without singular kernel, Therm. Sci., 20 (2016), no. $3,871-877.1$

[20] J.-H. He, S. K. Elagan and Z. B. Li, Geometrical explanation of the fractional complex transform and derivative chain rule for fractional calculus, Phys. Lett., A 376 (2012), no. 4, 257-259. 1

[21] J.-H. He and Y. Hu, On fractals space-time and fractional calculus, Thermal Science, 20 (2016), no. 3, 773-777. 1

[22] J.-H. He and Z. B. Li, Converting fractional differential equations into partial differential equations, Thermal Science, 16 (2012), no. 2, 331-334. 1

[23] J. Hristov, Transient heat diffusion with a non-singular fading memory: from the Cattaneo constitutive equation with Jeffrey's kernel to the Caputo-Fabrizio time fractional derivative, Therm. Sci., 20 (2016), 765-770. 1

[24] A.A. Kilbas, H.M. Srivastava and J.J. Trujillo, Theory and applications of fractional differential equations, Elsevier Science B.V., Amsterdam, the Netherlands, 204, 2006. 1

[25] Z.-B. Li and J.-H. He, Fractional complex transform for fractional differential equations, Math. Comput. Appl., 15 (2010), no. 5, 970-973. 1

[26] H.-Y. Liu, J.-H. He and Z.-B. Li, Fractional calculus for nanoscale flow and heat transfer, Internat. J. Numer. Methods Heat Fluid Flow, 24 (2014), no. 6, 1227-1250. 1

[27] J. Losada and J.J. Nieto, Properties of the new fractional derivative without singular Kernel. Progr. Fract. Differ. Appl., 1 (2015), no. 2, 87-92. 1

[28] A. Rachah and DFM. Torres, Mathematical modelling, simulation, and optimal control of the 2014 Ebola outbreak in West Africa, Discrete Dyn. Nat. Soc., 2015 (2015), Art. ID 842792. 1

[29] K. Razminia, A. Razminia and D. F. M. Torres, Pressure responses of a vertically hydraulic fractured well in a reservoir with fractal structure, Appl. Math. Comput., 257 (2015), 374-380. 1

[30] S. Rosa and D. F. M. Torres, Optimal control of a fractional order epidemic model with application to human respiratory syncytial virus infection, Chaos Solitons Fractals, 117 (2018), 142-149. 1

[31] A. B. Salati, M. Shamsi and D. F. M. Torres, Direct transcription methods based on fractional integral approximation formulas for solving nonlinear fractional optimal control problems, Commun. Nonlinear Sci. Numer. Simul., 67 (2019), 334-350. 1

[32] M.R. Sidi Ammi, M. Tahiri and D.F.M. Torres, Global stability of a Caputo fractional SIRS model with general incidence rate, Mathematics in Computer Science, (2021), 1-15. 1, 3

[33] K.-L. Wang and S. Y. Liu, He's fractional derivative and its application for fractional Fornberg-Whitham equation, Thermal Science, 21 (2017), no. 5, 2049-2055. 1

[34] W. Wojtak, C. J. Silva and D. F. M. Torres, Uniform asymptotic stability of a fractional tuberculosis model, Math. Model. Nat. Phenom., 13 (2018), no. 1, Art. 9, 10 pp. 1

[35] X.-E. Wu and Y.-S. Liang, Relationship between fractal dimensions and fractional calculus, Nonlinear Sci. Lett., A 8 (2017), no. $1,77-89.1$

[36] X. Wu, L. Tang and T. Zhong, An iteration algorithm for fractal dimensions of a self-similar set, Nonlinear Sci. Lett., A 8 (2017), no. 1, 117-120. 1

[37] X. Zhang and X. Liu, Backward bifurcation of an epidemic model with saturated treatment function, J. Math. Anal. Appl., 348 (2008), 433-443. 3

[38] X.-J. Yang, Advanced local fractional calculus and its applications, World Science, New York, 2012. 1

[39] X.-J. Yang, J. A. T. Machado and D. Baleanu, Exact traveling-wave solution for local fractional Boussinesq equation in fractal domain, Fractals, 25 (2017), no. 4, 1740006, 7 pp. 1

[40] X.-J. Yang , HM. Srivastava and JAT. Machado, A new fractional derivative without singular kernel: application to the modelling of the steady heat flow, Therm. Sci., 20 (2016), 753-756. 1

[41] X.-J. Yang, ZZ. Zhang and HM. Srivastava, Some new applications for heat and fluid flows via fractional derivatives without singular kernel, Therm. Sci., 20 (2016), 833-839. 1

[42] Yuan Qing and B. E. Rhoades, T-Stability of Picard iteration in metric spaces. Hindawi Publishing Corporation Fixed Point Theory and Applications, 2008, Article ID 418971, 4 pp. 4.1, 4.2, 4.3 